\title{
THE EFFECT OF POLYANILINE CONTENT DEPOSITED ON SILICA GEL PARTICLES ON THE ELECTRORHEOLOGY OF SILICONE-OIL SUSPENSIONS
}

\author{
V. PAVLÍNEK AND P. SÁHA \\ Tomas Bata University in Zlin, Faculty of Technology, 76272 Zlin, Czech Republic \\ E-mail:pavlinek@ft.utb.cz
}

T. KITANO

National Institute of Advanced Industrial Science and Technology, Macromolecular Technology Research Center, Tsukuba 305-8565, Japan

J. HROMÁDKOVÁ, J. STEJSKAL AND O. QUADRAT

Institute of Macromolecular Chemistry, Academy of Sciences of the Czech Republic, 16206 Prague 6, Czech Republic

\begin{abstract}
Investigation of the electrorheological effect of silicone-oil suspensions of silica particles coated with polyaniline base in a DC electric field revealed that breaking stress, as a criterion the intensity of the electrorheological phenomenon, steeply increased at first with coating thickness. At relatively low polyaniline content (volume fraction $\approx 0.05$ ), it has reached a value several times higher than that with suspension of pure silica. Then they became virtually constant or slightly increased. The frequency spectra of dielectric characteristics of these systems reflect high relaxation times. The results suggest that the interfacial polarization of particles is predominantly controlled by polarizability of their surface layer, and the influence of the thickness is of secondary importance.
\end{abstract}

\section{Introduction}

The electrorheological (ER) effect is defined as a reversible change in the structure of a suspension composed of solid particles dispersed in liquid medium after application of an electric field. It has been studied for many years and has been the object of many studies and reviews [1-6]. The polarized particles dispersed in a non-conducting medium create chains or columns oriented in the electric-field direction. They have a much higher resistance against deformation than randomly dispersed particles in a field-off suspension. In many cases, polarization forces are so high that a yield stress of the fluid appears. i.e. the material displays a solid-like structure. Due to shear forces such structure may be destroyed at high shear rates and, consequently, the apparent viscosity of suspension decreases to a value observed in the absence of electric field.

Naturally, it was tempting to utilize this phenomenon in practice in a variety of technical applications, such as clutches, torque transducers, dampers, etc. However, there are still great difficulties in practical engineering due to insufficient performance of the fluid studied so far.

Many powdered materials, both organic and inorganic, have been proposed as suspension particles. Among them, semi-conducting polymers, such as polyaniline (PANI), polypyrrole, polythiophene, poly-p-phenylene and various copolymers have been shown to be most promising [7-20]. Especially the structured particles with different core/shell dielectric properties have been objects of recent investigation [21-23]. In our previous study [22] of various silicas coated with PANI, we have found that the polarizability of particles is not significantly affected by the shape and size of the core. 
On the other hand, the ER effect considerably increased after the particles were coated with a thin layer of PANI. It is of interest if the ER behaviour would be affected by the thickness of PANI overlayer. The elucidation of this problem is an object of this study.

\section{Experimental}

\subsection{Coating of silica particles with polyaniline base}

Five samples of silica particles (Ultrasil VN 3, Degussa-Hüls, Germany; average particle size of $18 \mu \mathrm{m}$ ) coated with a layer of PANI base have been prepared by the oxidative polymerization of aniline [24] and subsequent deprotonation. To various amount of silica, $1-30 \mathrm{~g}$, a $100 \mathrm{ml}$ of reaction mixture containing $0.2 \mathrm{M}$ aniline hydrochloride and 0.25 ammonium peroxydisulfate [25] has been added at $20^{\circ} \mathrm{C}$. This volume of reaction mixture generates $c a .2 \mathrm{~g}$ of PANI hydrochloride and, by varying the silica gel content, the various weight fractions of PANI in the composite could be achieved (Table 1). Next day, the PANI-coated particles were separated on a filter and PANI salt deprotonated with an excess of $1 \mathrm{M}$ ammonium hydroxide to the corresponding PANI base. After washing with acetone, the particles coated with PANI base were dried in air and then in vacuo at $60^{\circ} \mathrm{C}$.

Table 1. Silica gel particles coated with a PANI base

\begin{tabular}{lccccccc}
\hline Sample code & S1 & S2 & S3 & S4 & S5 & S6 & S7 \\
\hline Content of PANI (wt. \%): & 0 & 5.7 & 8.3 & 15 & 37 & 64.3 & 100 \\
\hline
\end{tabular}

\subsection{Particle characterization}

The silica particles were porous and had an apparent density $\rho_{\mathrm{s}} \approx 0.8 \mathrm{~g} \mathrm{~cm}^{-3}$. Considering the average size of silica particles, $\mathrm{D} \sim 18 \mu \mathrm{m}$, and the density of PANI base [25], $\rho_{\mathrm{PANI}}=1.24 \mathrm{~g} \mathrm{~cm}^{-3}$, the thickness of PANI layer would range from 0.1 to $2.2 \mu \mathrm{m}$ provided that all PANI had been deposited as a film on the surface of silica particles.

The micrographs (Fig. 1) obtained with optical microscopy (Zetopan Pol, Reichert, Austria) demonstrate the uniform coating of silica, except for sample S7 where large particles of PANI powder were present.

\subsection{Suspension preparation}

Two series of $11.8 \mathrm{vol}$ \% suspensions of silica particles in silicone oil (Fluid 200, Dow Corning, UK; viscosity $\eta_{c}=108 \mathrm{mPa} \mathrm{s}$, density $\rho_{c}=0.965 \mathrm{~g} \mathrm{~cm}^{-3}$ ) have been prepared and their ER behaviour compared. The series number one was represented by suspensions of PANI-coated particles having various PANI content. In the series number two, silica and PANI powder were mixed in various proportions. 


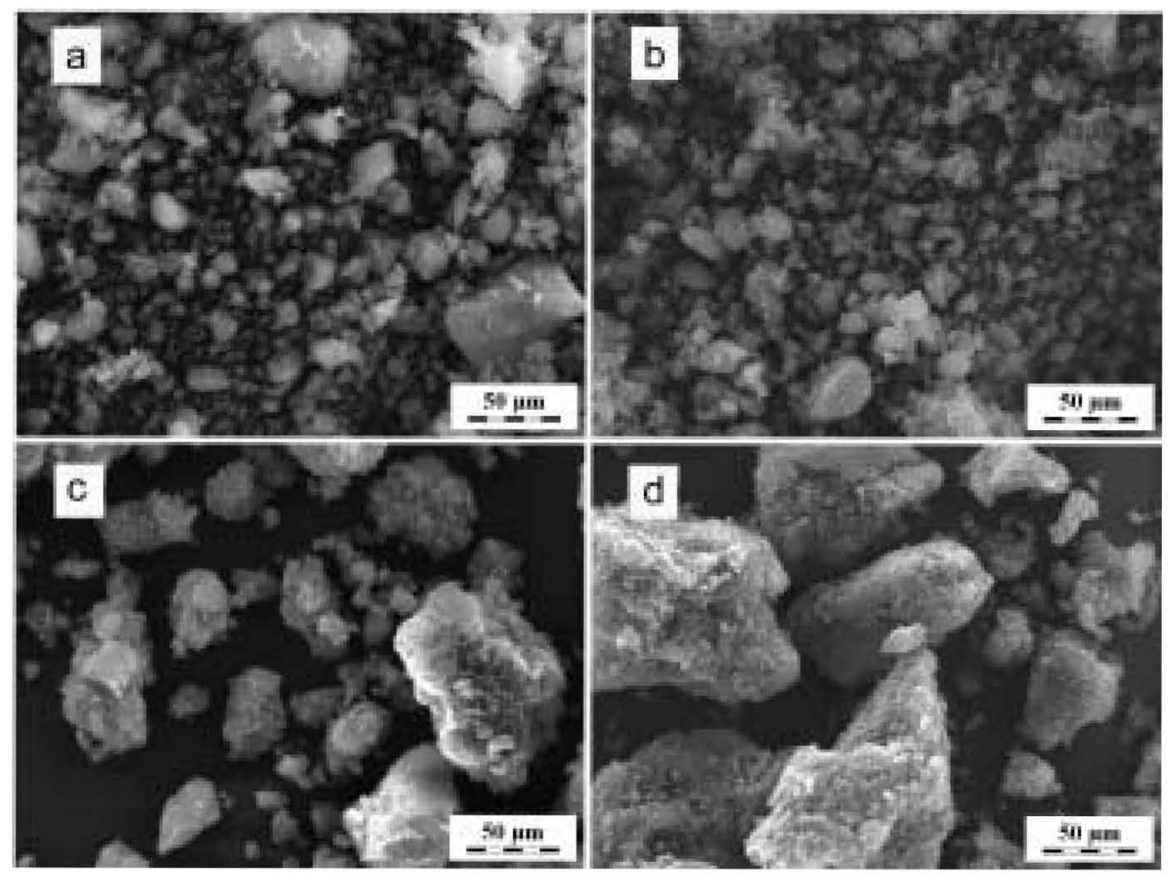

Figure 1. Micrographs of the neat and PANI-coated silica particles. Content of PANI ( wt. \%): (a) 0 , (b) 5.7, (c) 64.3 , (d) 100 .

\subsection{Electrorheology}

Measurements of rheological properties were carried out at $20^{\circ} \mathrm{C}$ using a Bohlin Gemini coaxial cylinder viscometer (Bohlin Instruments, UK). The suspensions were placed in the Couette cell with a rotating inner cylinder of $14 \mathrm{~mm}$ in diameter and the outer cylinder separated by a $0.7 \mathrm{~mm}$ gap. They were connected to a DC power supply (TREK 668B, TREK, USA) producing a field strength $\mathrm{E}=0.5-2 \mathrm{kV} \mathrm{mm}^{-1}$. The suspensions were mechanically stirred before each ER measurement. Particle sedimentation during measurement was negligible.

\subsection{Dielectric measurement}

Dielectric properties involving the frequency spectra of relative permittivity, $\varepsilon^{\prime}$, and relative dielectric loss, $\varepsilon$ ", in the frequency range $10-10^{5}$ have been measured with Hioki 3522 RCL Tester, Hioki, Japan.

\section{Results}

The dielectric measurements showed that both relative permittivity and relative dielectric loss factor of PANI-coated particles suspensions increase with PANI content. The relaxation maxima on the $\varepsilon^{\prime \prime}-f$ plots of suspensions of coated particles occur at very low frequencies $(<10 \mathrm{~Hz})$ and with higher PANI content shift to the right. On the other 
hand in case of suspensions of PANI powder/silica mixtures the relaxation frequencies were alike and corresponded to the value of pure PANI sample. Depending on PANI content in the mixture the magnitude of the maxima on the frequency spectra of the relative dielectric loss factor incerased.

The comparison of the efficiency of investigated suspensions was performed using a breaking stress $\tau_{\mathrm{b}}$ obtained by controlled shear stress measurement (Fig. 2).

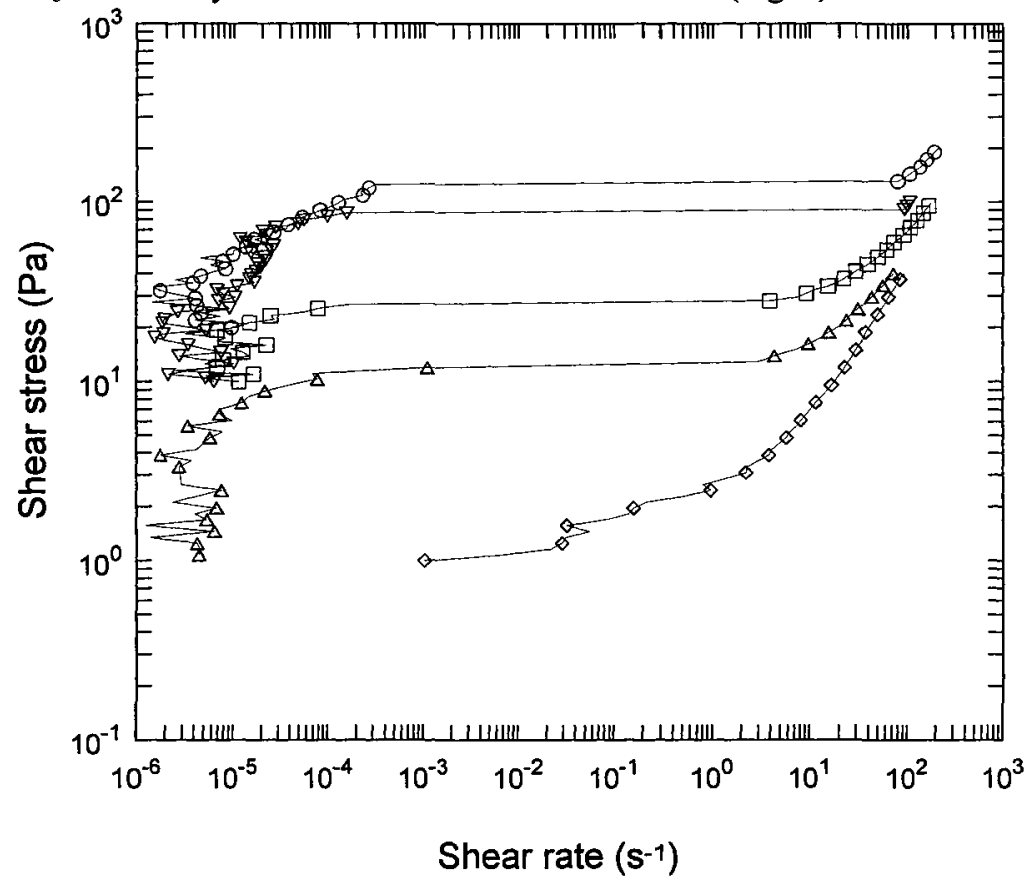

Figure 2. The dependence of the shear stress on the shear rate in controlled shear stress mode. Electric field strength $\mathrm{E}\left(\mathrm{kV} \mathrm{mm}^{-1}\right)$ : $\diamond 0, \triangle 0.5, \square 1, \nabla 1.5, \bigcirc 2$.

This quantity represents a maximum stress at which the particle chains formed in the electric field remain undestroyed by shear forces and characterizes the stiffness of particle chain-like structure more reliably than the dynamic yield stress evaluated through an extrapolation of the curly course of the shear stress to zero shear rate measured in the controlled shear rate mode (Fig. 3).

Figure 4 shows that breaking stress with the thickness of PANI particle layer at first steeply rose and, at relatively low PANI concentration (5.6 wt. \%), reached a value several times higher than that of uncoated silica suspension. Then, after a small decrease it slightly increased to a value corresponding to a suspension of neat PANI or remained virtually unchanged depending on voltage applied. In contrast, in case of the suspensions of PANI powder/silica mixtures the breaking stress is significantly lower and only continuously increases with the PANI content. It is clear that a strong ER effect of PANI coated silica particles with relatively low PANI amount as a consequence of interfacial polarization sets in and further increase in the thickness of the particle coating affects the ER properties negligible. 


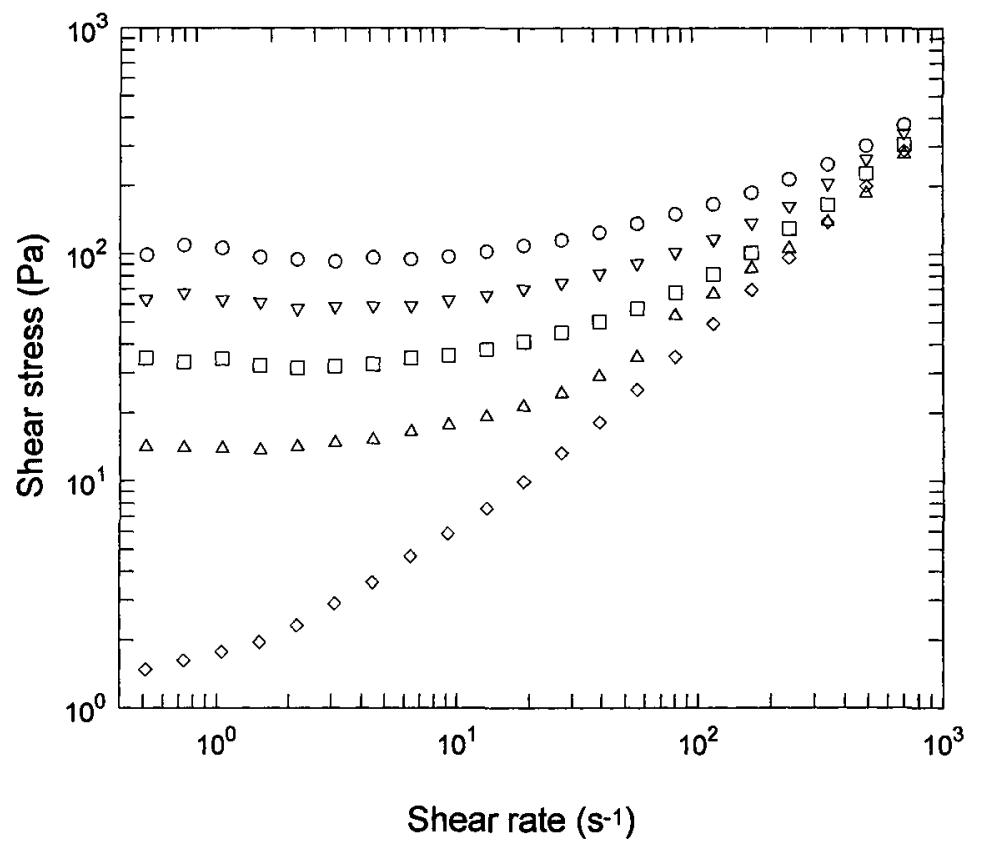

Figure 3. The dependence of the shear stress on the shear rate in controlled shear rate mode. Points denoted as in Figure 2.

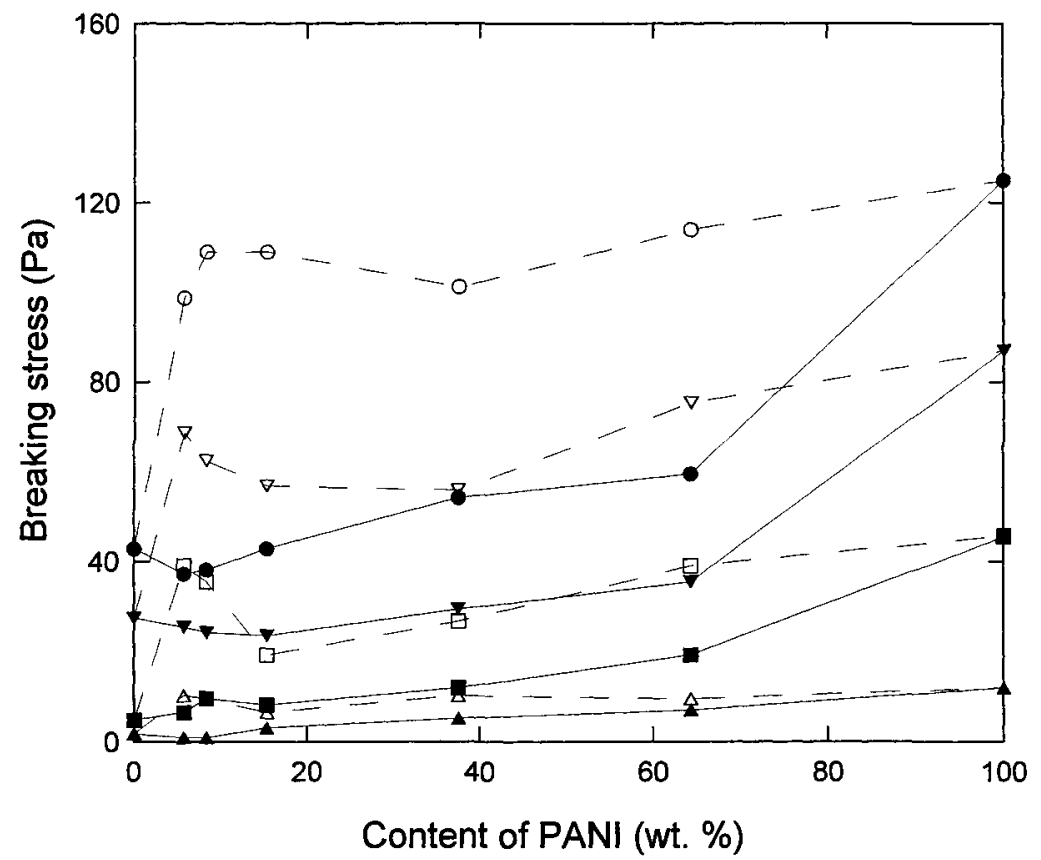

Figure 4. The dependence of the breaking stress $\tau_{0}$ on the PANI content in coated particles (open points) and PANI content in the mixtures with silica (solid points). Electric field strength denoted as in Figure 2. 


\section{Discussion and conclusion}

It is generally accepted that the ER effect is closely related to polarization mechanism in suspension particles. The results obtained so far indicate that an appreciable ER effect occurs when the relaxation frequency, at which the relative dielectric loss factor $\varepsilon^{\prime \prime}$ has a local maximum, is in the frequency range $10-10^{5} \mathrm{~Hz}$ corresponding to the interfacial polarization $[2,26-28]$. The magnitude of polarization depends on the difference of dielectric constants $\varepsilon^{\prime}$ below and above this frequency value. Besides, conductivity of suspension particles should be not very low [28].

An initial increase of ER efficiency of silica particles due to PANI coating is easy to understand. On the other hand, however, the results revealed that thickness of the PANI layer affects the ER properties negligibly or only very little. It is clear that this behaviour is a consequence of the mechanism of interfacial polarization of particles controlled by charge carriers migration predominantly on the particle surface or in the double layer.

\section{Acknowledgements}

The authors wish to thank to Ministry of Education of the Czech Republic (MSM 265200015 and KONTAKT ME 564) and the Grant Agency of the Academy of Sciences of the Czech Republic (A 4050313) for financial support.

\section{References}

1. Block H. and Kelly J. P., Electro-rheology. J. Phys. D: Apl. Phys. 21 (1988) pp. 1661-1667.

2. Block H., Kelly J. P. Quin A. and Watson T., Materials and mechanism in electrorheology, Langmuir 6 (1990) pp. 6-14.

3. Hao T., Electrorheological fluids, Adv. Mater. 13 (2001) pp. 1847-1857.

4. Hao T., Electrorheological suspensions, Adv. Colloid Interface Sci. 97 (2002) pp. 135.

5. Jordan T. C. and Shaw M. T., Electrorheology, IEEE Trans. Electric Insul. 24 (1989) pp. 849-878

6. Parthasarathy M. and Klingberg D.J., Electrorheology: mechanisms and models, Mater. Sci. Eng. $R 17$ (1996) pp. 57-103.

7. Cho M. S., Choi H. J. and To K., Effect of ionic pendant groups on a polyanilinebased electrorheological fluid, Macromol. Rapid Commun. 19 (1998) pp. 271-733.

8. Cho H. J., Kim J. W., Choi H. J., Weber R. M. and Jhon M. S., Electrorheological characteristics of polyaniline and its copolymer suspensions with ionic and nonionic substituents, Colloid Polym. Sci. 278 (2000) pp. 61-54.

9. Cho C. H., Choi H. J., Kim J. W. and Jhon M. S., Synthesis and electrorheology of aniline-pyrrole copolymer, J. Mater. Sci. 39 (2004) pp. 1883-1885.

10. Choi H. J., Kim T. W., Cho M. S., Kim S. G. and Jhon M. S., Electrorheological characterization of polyaniline dispersions, Eur. Polym. J. 33 (1997) pp. 699-703.

11. Choi H. J., Cho M. S. and To K., Electrorheological and dielectric characteristics of semiconductive polyaniline-silicone oil suspensions, Physica A 254 (1998) pp. 272279.

12. Choi H. J., Lee J. H., Cho M. S. and Jhon M. S., Electrorheological characterization of semiconducting polyaniline suspensions, Polym. Eng. Sci. 39 (1999) pp. 493-499. 
13. Choi H. J., Kim J. W. and To K., Electrorheological characteristics of semiconducting poly(aniline-co-o-ethoxyaniline) suspension, Polymer 40 (1999) pp. 2163-2166.

14. Choi H. J., Kim J. W. and To K., Synthesis and electrorheological behaviour of semiconducting poly(aniline-co-c-ethoxyaniline), Synth. Met. 101 (1999) pp. 697698.

15. Gow C. J. and Zukoski IV C. F., The electrorheological properties of polyaniline suspensions, J. Colloid Interface Sci. 136 (1990) pp. 175-188.

16. Jang W. H., Kim J. W., Choi H. J. and Jhon M. S., Synthesis and electrorheology of camphorsulfonic acid doped polyaniline suspensions, Colloid Polym. Sci. 279 (2001) pp. 823-827.

17. Kim Y. D. and Park D. H., The electrorheological responses of suspensions of polypyrrole-coated polyethylene particles, Colloid Polym. Sci. 142 (2002) pp. 828 $-834$.

18. Sohn J., Sung J.H., Choi H. J. and Jhon M. S., The effect of particle concentration of poly(p-phenylene) on electrorheological response, J.Appl.Polym. Sci. 84 (2002) pp.2397-2403.

19. Plocharski J., Rozanski M. and Wycislik H., Electrorheological effect in suspensions of conductive polymers, Synth. Met. 102 (1999) pp. 1354-1357

20. Xie H. Q. and Guan J. G., Study on electrorheological properties of semiconducting polyaniline-based suspensions, Angew. Makromol. Chem. 235 (1996) pp. 21-37.

21. Kuramoto N., Yamazaki M., Nagai K., Koyama K., Tanaka K., Yatsuzaka K. and Higashiyama $Y$., The electrorheological property of polyaniline-coated copolystyrene particle suspensions, Rheol. Acta 34 (1995) pp. 298-302.

22. Lengálová A., Pavlínek V., Sáha P., Stejskal J., Kitano T., Quadrat O., The effect of dielectric properties on the electrorheology of suspensions of silica particles coated with polyaniline, Physica A 321 (2003) 411-424.

23. Ly J. and Zhao X., Electrorheological properties of a polyaniline-montmorillonite clay nanocomposite suspension, J. Mater. Chem. 12 (2002) pp. 2603-2605.

24. Stejskal J. and Gilbert R.G., Polyaniline. Preparation of a conducting polymer, Pure Appl. Chem. 74 (2002) pp. 857-867.

25. Stejskal J., Trchová M., Fedorova S. and Sapurina I., Surface polymerization of aniline on silica gel, Langmuir 19 (2003) pp. 3013-3018.

26. Hao T., Xu Z. and Xu Y., Correlation of the dielectric properties of dispersed particles with the electrorheological effect, J. Colloid Interface Sci. 190 (1997) pp. 334-340.

27. Hao T., Kawai A. and Ikazaki F., Mechanism of the electrorheological effect: Evidence from the conductive, dielectric, and surface characteristics of water-free electrorheological fluids, Langmuir 14 (1998) pp. 1256-1262.

28. Ikazaki F., Kawai A., Uchida., Kawakami T., Edamura K., Sakurai K., Anzai H. and Asako Y., Mechanism of electrorheology: the effect of the dielectric property, $J$. Phys. D: Appl.Phys. 31 (1998) pp. 336-347. 
Copyright of International Journal of Modern Physics B: Condensed Matter Physics is the property of World Scientific Publishing Company and its content may not be copied or emailed to multiple sites or posted to a listserv without the copyright holder's express written permission. However, users may print, download, or email articles for individual use. 